\title{
3D Printing of Highly Stretchable and Sensitive Strain Sensors Using Graphene Based Composites ${ }^{\dagger}$
}

\author{
Meshari Alsharari ${ }^{1,2,3}$, Baixin Chen ${ }^{1}$ and Wenmiao Shu ${ }^{2, *}$ \\ 1 School of Engineering and Physical Sciences, Heriot-Watt University, Edinburgh EH14 4AS, UK; \\ mma57@hw.ac.uk (M.A.); b.chen@hw.ac.uk (B.C.) \\ 2 Department of Biomedical Engineering, University of Strathclyde, Glasgow G4 0NW, UK \\ 3 Department of Engineering, Jouf University, Sakaka 72388, Saudi Arabia \\ * Correspondence: will.shu@strath.ac.uk; Tel.: +44-141-548-3031 \\ + Presented at the Eurosensors 2018 Conference, Graz, Austria, 9-12 September 2018.
}

Published: 21 December 2018

\begin{abstract}
In this research, we present the development of 3D printed, highly stretchable and sensitive strain sensors using Graphene based composites. Graphene, a 2D material with unique electrical and piezoresistive properties, has already been used to create highly sensitive strain sensors. In this new study, by co-printing Graphene based Polylactic acid (PLA) with thermoplastic polyurethane (TPU), a highly stretchable and sensitive strain sensor based on Graphene composites can be 3D printed for the first time in strain sensors. The fabrication process of all materials is fully compatible with fused deposition modeling (FDM) based 3D printing method, which makes it possible to rapidly prototype and manufacture highly stretchable and sensitive strain sensors. The mechanical properties, electrical properties, sensitivity of the 3D printed sensors will be presented.
\end{abstract}

Keywords: 3D printing; strain sensors; stretchable; Graphene

\section{Introduction}

Highly sensitive and stretchable sensors have wide potential applications in soft robotics, flexible/wearable electronics and prosthetics [1,2]. Additive manufacturing by means of 3D printing is a promising technology that has the advantages of rapid and distinct iteration of prototyping manufacturing [2]. 3D printable Graphene filaments, though very sensitive, have poor mechanical properties and hence are very limited in its stretchability or elasticity $[3,4]$. For strain sensing applications, PLA has strong bonding with Graphene and ease of 3D printability, however; its rigidity limits the stretchability [4]. On the other hand, thermoplastic polyurenthene (TPU) is stretchable and printable material, but has an inherent negative strain effect due to the poison ratio effect, which limits the sensitivity of any stretchable sensors [5,6].

We here proposed to develop a Graphene based PLA with TPU successfully compounded by melt processing and simultaneously 3D co-printed, for the creation of highly stretchable and sensitive strain sensors. The current 3D printed Graphene based sensors suffer poor stretchability reported up to $8 \%$ strain [3,4]. Herein, the co-printing of Graphene/PLA/TPU significantly enhanced the stretchability, while keeping the sensitivity of a typical Graphene based strain sensors. 


\section{Materials and Methods}

\subsection{Materials}

The materials used in this research include conductive Graphene PLA pellets, purchased from Graphene3D lab with electrical conductivity of $0.6 \mathrm{~S} / \mathrm{cm}$ and stretchable TPU filament with shore hardness of 85A was purchased from NinjaTek Ltd. (Manheim, PA, USA).

\subsection{Composites Preparation}

For the sensing material, Graphene PLA "GP" pellets were first dry mixed with the addition of TPU pellets and then hot blended in a single-screw extruder (Noztek Pro, Noztek Ltd., West Sussex, UK) to form a strand of $1.75 \mathrm{~mm}$ of diameter as shown in Figure 1a to be later employed for the 3D printing process [Figure 1]. The mass loading (wt \%) of the TPU added to GP is denoted as GP- $x$ TPU, where $x$ represent the mass loading wt $\%$ of TPU to GP. For the substrate, the TPU filament was used as received.

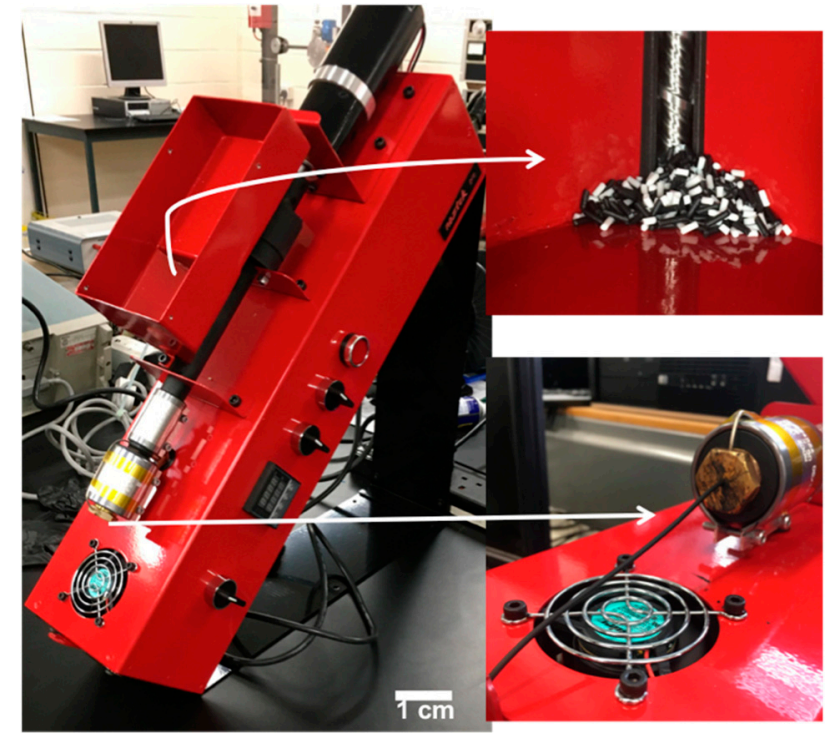

(a)

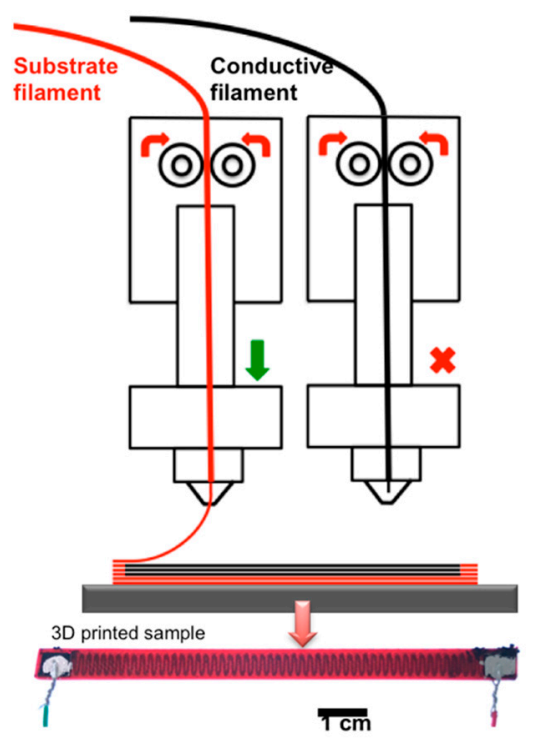

(b)

Figure 1. (a) Preparation of the sensing material via single-screw extruder (inset of mixed pellets and the extruded $1.75 \mathrm{~mm}$ strand); (b) The schematic process of 3D FDM dual printing.

\subsection{D Fabrication of the Strain Sensors}

For the 3D printing process, a Flashforge Creator Pro FDM printer (FlashForge Corporation, Jinhua, Zhejiang, China), with dual extrusion system was used to fabricate the strain sensors reported here. Three samples with mass loadings of GP, GP-10TPU and GP-20TPU were prepared. The prepared sensing filament illustrated in Figure 1b, was simultaneously printed in meandering sine wave like structure embedded within an insulating pure TPU substrate [7]. The 3D printed samples as illustrated in Figure $1 \mathrm{~b}$ measure $100 \times 6.6 \times 2 \mathrm{~mm}$ and Table 1 shows the 3D printing parameters.

Table 1. FDM parameters used for 3D printing of strain sensors.

\begin{tabular}{cc}
\hline Parameter & Value \\
\hline Print Temperature $\left({ }^{\circ} \mathrm{C}\right)$ & 230 \\
Platform Temperature $\left({ }^{\circ} \mathrm{C}\right)$ & 60 \\
Print Nozzle Diameter $(\mathrm{mm})$ & 0.4 \\
Layer Height $(\mathrm{mm})$ & 0.2 \\
Print Infill $(\%)$ & 100 \\
Print Speed $(\mathrm{mm} / \mathrm{s})$ & 15 \\
\hline
\end{tabular}




\section{Results}

\subsection{Electromechanical Properties}

In order to evaluate the electromechanical response of the 3D printed sensors, each sensor was first wired and silver coated at the two ends to reduce the contact resistance to the probes. The samples were connected to a Picolog Data Logger with a constant voltage of $2.5 \mathrm{~V}$ in order to measure the electrical resistance. They were also attached to an Instron 3367 (Instron Ltd., Norwood, MA, USA) machine to perform the tensile test at a strain rate of $4 \mathrm{~mm} / \mathrm{s}$. To evaluate the sensitivity of the strain sensors, the relative change in resistance was expressed as $\Delta R / R_{0}$ (where $\Delta R$ represents the change in resistance during strain loading and $R_{0}$ represents the value of the original sensor's resistance). Figure 2a shows the first response of change in resistance loaded to $100 \%$ strain of samples with GP, GP-10TPU and GP-20TPU.

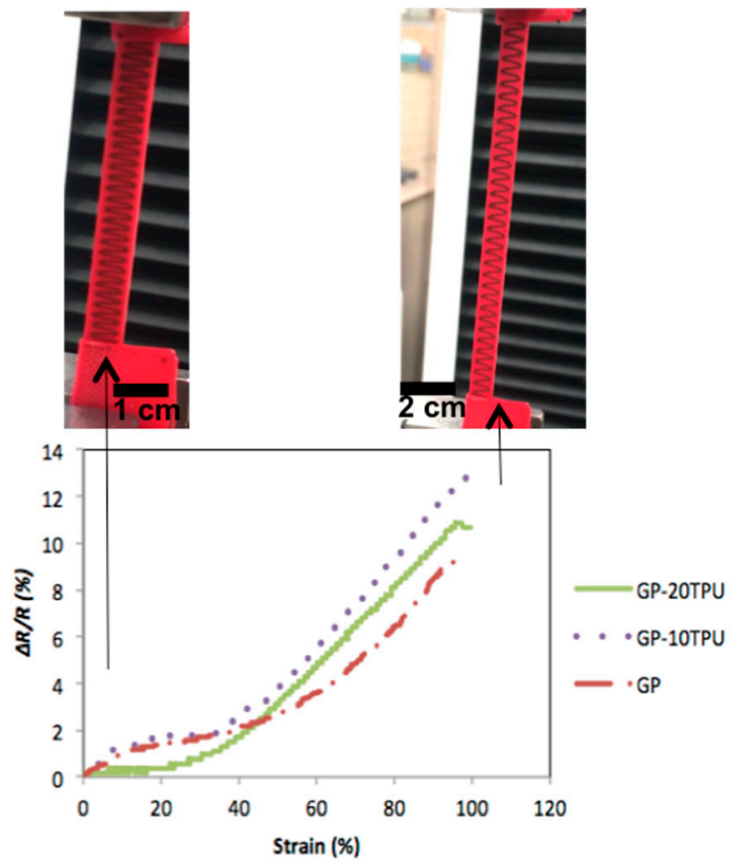

(a)

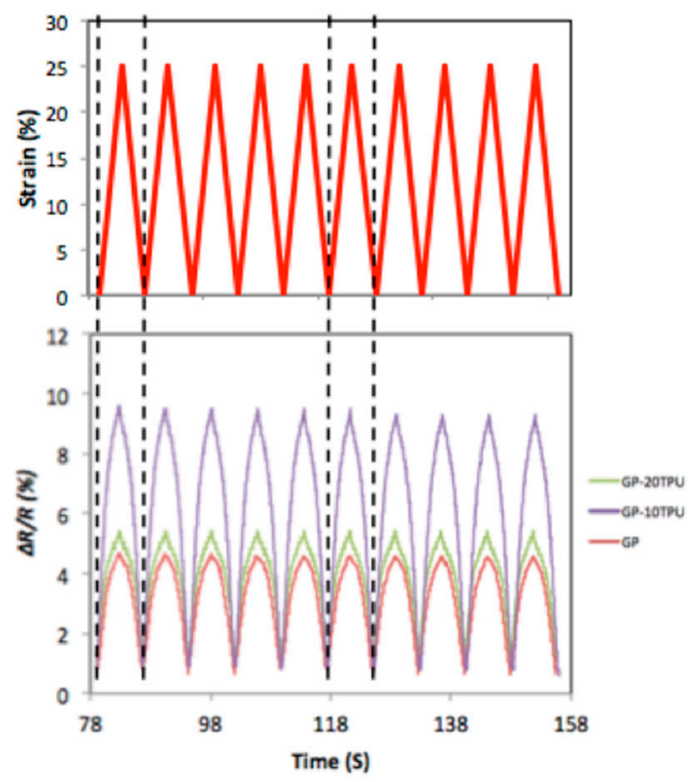

(c)

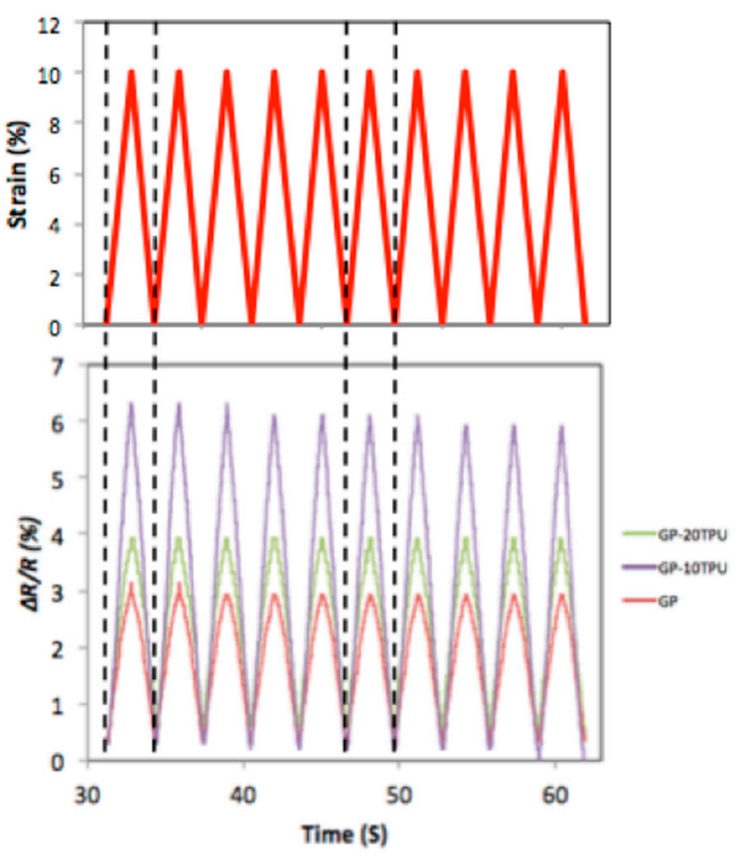

(b)

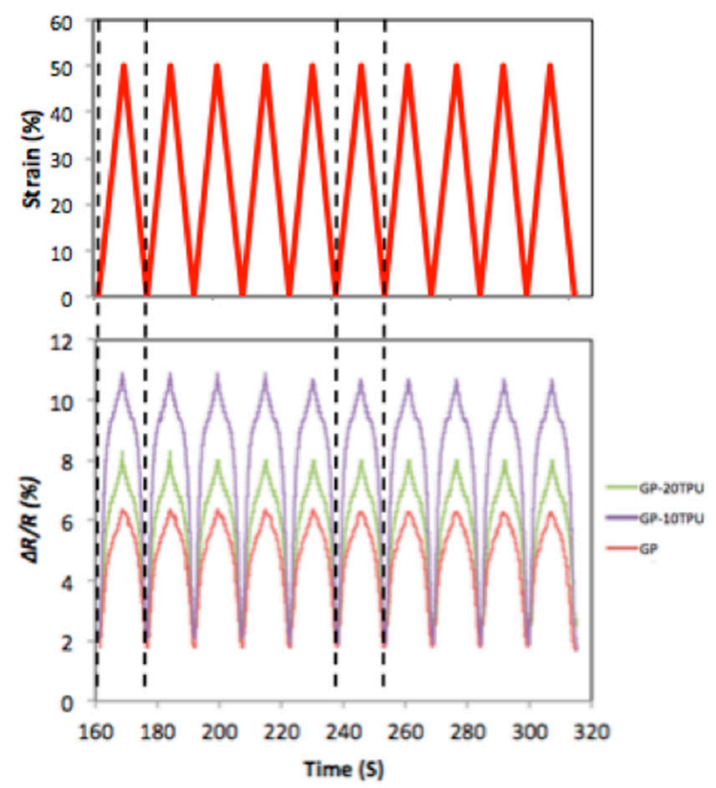

(d)

Figure 2. (a) First response of change in resistance at 100\% strain (inset of the sample at $0 \& 100 \%$ strain); (b) cyclic loading at 10\% strain; (c) cyclic loading at 25\% Strain; (d) cyclic loading at 50\% strain. 
It was observed that all of the three samples were able to withstand a peak first cycle of $100 \%$ strain due to the meander structure of the sensing pattern embedded in the stretchable TPU matrix as shown in Figure 2a, and were found to perform steady change in resistance. Also, the samples, which had the addition of stretchable TPU, showed higher sensitivity than of neat GP (See Figure 2a).

\subsection{Cyclic Strain Behaviour}

To further examine the 3D printed samples, cyclic strains of $10 \%, 25 \%$ and $50 \%$ were performed and illustrated in Figure $2 \mathrm{~b}-\mathrm{d}$ respectively, of cycles between 11 th to 20th cycles. The samples showed gradual increase of change in resistance with strain loading and decrease with unloading, which is described as positive strain effect [5]. It was also observed that the addition of TPU to GP enhanced the sensitivity of the strain sensors, which can be confirmed in all of the cyclic strains shown in Figure $2 b-d$. In addition, the peak pre straining of $100 \%$ strain at the first cycle resulted the strain sensors to perform with good sensing stability at lower cyclic strains, represented with good recoverability and reproducibility [8].

\section{Conclusions}

In summary, a simple method of 3D printed conductive composites simultaneously embedded within a stretchable substrate was presented. The electromechanical and cyclic loading of the samples were tested and showed their feasibility as strain sensors. The co-printing of Graphene/PLA/TPU in a meander sine wave like structure significantly enhanced the stretchability by up to 4 folds, to over $30 \%$ strain, while keeping the sensitivity of a typical Graphene based strain sensors. The cyclic loading and unloading of the printed samples showed good sensing stability and recoverability, while maintaining positive strain effect.

\section{References}

1. Berman, B. 3-D printing: the new industrial revolution. Bus. Horiz. 2012, 55, 155-162.

2. MacDonald, E.; Salas, R.; Espalin D.; Perez, M.; Aguilera, E.; Muse, D.; Wicker, R.B. 3D printing for the rapid prototyping of structural electronics. IEEE Access 2014, 2, 234-242.

3. Zhang, D.; Chi, B.; Li, B.; Gao, Z.; Du, Y.; Guo, J.; Wei, J. Fabrication of highly conductive graphene flexible circuits by 3D printing. Synth. Met. 2016, 217, 79-86.

4. Jesse, G.; Travis F. 3D Printed Strain Gauge Geometry and Orientation for Embedded Sensing. In Proceedings of the 58th AIAA/ASCE/AHS/ASC Structures, Structural Dynamics, and Materials Conference, Grapevine, TX, USA, 9-13 January 2017; (AIAA 2017-0350); AIAA SciTech Forum: Reston, VA, USA, 2017.

5. Christ, J.F.; Aliheidari N.; Ameli, A.; Pötschke, P. 3D printed highly elastic strain sensors of multiwalled carbon nanotube/thermoplastic polyurethane nanocomposites. Mater. Des. 2017, 131, 394-401.

6. Lozano-Pérez, C.; Cauich-Rodríguez, J.V.; Avilés, F. Influence of rigid segment and carbon nanotube concentration on the cyclic piezoresistive and hysteretic behavior of multiwall carbon nanotube/segmented polyurethane composites. Compos. Sci. Technol. 2016, 128, 25-32.

7. Balakrisnan, B.; Nacev, A.; Burke, J.M.; Dasgupta, A.; Smela, E. Design of compliant meanders for applications in MEMS, actuators, and flexible electronics. Smart Mater. Struct. 2012, 21, 075033.

8. Yazdani, H.; Hatami, K.; Khorsravi E.; Harper, K.; Grady, B.P. Strain-Sensitive conductivity of carbon black-filled PVC composites subjected to cyclic loading. Carbon 2014, 79, 393-405.

(C) 2018 by the authors. Licensee MDPI, Basel, Switzerland. This article is an open access article distributed under the terms and conditions of the Creative Commons Attribution (CC BY) license (http://creativecommons.org/licenses/by/4.0/). 